\title{
NON-FERMI LIQUID EFFECTS IN THE LOW TEMPERATURE BEHAVIOUR OF A DISORDERED STRONGLY INTERACTING ELECTRON SYSTEM
}

\author{
W.N. Huang and J.W. Rasul \\ Physics Department, University of Michigan, Ann Arbor, MI 48109-1120, USA \\ (Received 19 January 1993; in revised form 3 May 1993 by A.H. MacDonald
}

\begin{abstract}
We examine a version of the $U=\infty$ Hubbard model for which a $1 / N$ expansion can be carried out ( $N$ being the orbital degeneracy), in the presence of disorder. We calculate the $1 / N$ corrections to the partition function and single particle decay rate, treating the disorder by summing up those ladder diagrams in the impurity potential that are known to yield the diffusion pole in conventional disorder theories. We find, away from the critical metal-insulator filling, agreement with earlier weak interaction theories with a single parameter characterising the effective interaction, while close to the critical filling we find novel power law corrections whose origin may be traced to the strong momentum dependence of the diffusion pole in the density correlation function.
\end{abstract}

STRONGLY correlated electron systems are extremely sensitive to disorder. Heavy fermion systems show huge changes in the residual resistivity regardless of whether the alloy substitution is taking place on the normal host or the rare earth sites [1]. In high $T_{c}$ systems a small amount of impurities can drive a transition directly from the superconducting to the insulating phase [2].

Theoretically, most work has started from the disordered weakly localized system, and incorporated the effects of switching on electron interactions in the diffusive regime [3]. More recently scaling approaches have been applied to the problem [4], but from a microscopic point of view, the aim has basically been to incorporate interaction effects into the very thorough field theoretical treatment that describes the Anderson transition [5-7].

Alternatively, one can start from the strongly correlated electron systems and assess the effects of including disorder. For heavy fermion systems a number of authors have taken this approach, starting with the mean field large- $N$ limit of the Anderson lattice and then either treating the disorder effects within the CPA (Coherent Potential Approximation) [8] or by a block decimation procedure [9]. One of the authors [10] has even examined weak localization corrections for the case of impurity scattering of the conduction electrons.

Generally, these works have only included the strong correlation effects at the mean field level, where in the non-disordered limit the physics is that of a band of non-interacting quasiparticles. It is well known that to study quasiparticle interactions one has to examine Gaussian fluctuations around this mean-field picture. Such a formulation is necessary if one is to obtain either dynamic susceptibilities or selfenergy lifetime effects.

In the present paper we instigate such studies for a simpler model than the Anderson lattice, namely a version of the infinite $U$ Hubbard model for which a loop expansion can be carried out. We consider the $N$-fold degenerate infinite $U$ Hubbard model where the local electronic charge is scaled with the degeneracy [11]. At the mean field level this model reduces to a single band whose width is reduced by the Gutzwiller factor $1-n$, where $n$ is the electron filling. The loop expansion is then equivalent to including $1 / N$ corrections of the RPA type, involving particle-hole excitations, and we shall include the effects of weak disorder on these particle-hole terms by summing up those ladder graphs that are known to yield diffusive behaviour in the density correlation function.

We expect on the one hand to make contact with conventional "weakly interacting" theories [3] if the electron filling in the $U=\infty$ Hubbard model is not too close to $1 / N$, the critical value at which the insulating phase takes over. It is known that when electron interactions are involved one does not have to study transport properties alone in order to see the effects of disorder [3]. Bulk effects of the type mentioned earlier show non-Fermi liquid behaviour 
even when the disorder is only weak. By studying the one-loop RPA processes we hope to be able to understand how the effects of Gutzwiller projection combine with conventional disorder ideas.

A further reason for investigating this problem concerns the behaviour close to $1 / N$ filling. In the pure system the authors [12] found that the nature of the collective mode in the density fluctuation spectrum changes as this filling fraction is approached. Instead of "zero-sound" dispersion, the collective mode has a dispersion functionally identical to that of the bare electron motion in the model - if the electron bands are parabolic then so is the collective mode. It is found that the bare electron mass not the Gutzwiller renormalized mass enters this dispersion relation - the electrons can move as a whole in this way avoiding the infinite $U$ restriction.

In this paper we shall investigate the nature of this mode when disorder is present - we shall see that the usual diffusion pole behaviour is completely overwhelmed in the region close to $1 / N$ filling by a $q^{4}$ term. We shall see that this leads to a different behaviour in the self energy and low temperature specific heat compared to the conventional result. We should bear in mind, however, that the scaling of the local charge with degeneracy means that the Fermi wavevector scales with an inverse power of the degeneracy, so that this result may be special to the model under consideration.

Our starting point is the infinite $U$ Hubbard model for which the Lagrangian can be written

$$
\begin{aligned}
& L(\tau)=\sum_{i, j, m} f,^{+} m(\tau)\left[\left(\partial / \partial \tau-\mu+i \lambda_{j}(\tau)\right) \delta_{i j}\right. \\
& \left.\quad+t_{i j} \rho_{i} \rho_{j}\right] f_{j m}(\tau)+\sum_{j} i \lambda_{j}\left(\rho_{j}^{2}+\sum_{m} f_{j m}^{+} f_{j m}-Q\right)
\end{aligned}
$$

where $f_{i, m}$ denotes a fermion operator at site $i$ and in orbital $m, \mu$ denotes the chemical potential, $\lambda_{i}$ denotes a Lagrange multiplier, originally introduced to enforce the infinite $U$ constraint, and $\rho_{i}$ denotes the radial part of the Bose field that labels the empty site at site $i$. The quantity $Q$ is allowed to scale with the degeneracy $N$, and is taken to be unity at the end of the calculation. The standard gauge transformation [13] has been performed so that the Lagrange multiplier is now time dependent. To the above Lagrangian we add the following terms in the case of site disorder

$$
L_{i m p}(\tau)=\sum_{r_{i}} V\left(r_{i}\right) f_{i m}(\tau) f_{i m}(\tau)
$$

where an electron at site $r_{i}$ experiences a potential

$V\left(r_{i}\right)=\sum_{a} u\left(r_{i}-R_{a}\right)$

where $R_{a}$ denotes the set of impurity positions. The partition function is written in terms of the above as

$Z=\int D f D f D \rho D \lambda \exp \left[-\int_{0}^{\beta} d \tau\left(L(\tau)+L_{i m p}(\tau)\right)\right]$

where the site disorder term is absorbed into the constraint term $i \tilde{\lambda}_{j}(\tau)=i \lambda_{j}(\tau)+V\left(r_{j}\right)$. Integrating over the fermion fields yields the effective action

$$
\begin{aligned}
\tilde{L}(\tau)= & \sum_{i} i \lambda_{i}\left(\rho_{i}^{2}-Q\right)-N \operatorname{Tr} \ln \\
& {\left.\left[\partial / \partial \tau-\mu+i \tilde{\lambda}_{j}\right) \delta_{i j}+t_{i j} \rho i \rho j\right] }
\end{aligned}
$$

In the standard $1 / N$ expansion method we separate out from the effective action a mean field component in which the Bose fields are replaced by their saddle point values, determined by minimization of the free energy. This yields the mean field equations

$i \lambda_{0}=-\frac{N}{\beta} \sum_{\omega} \operatorname{Tr} t_{o j} G_{i j}(i \omega)$,
$\rho_{0}^{2}=Q-\frac{N}{\beta} \sum_{\omega} \operatorname{Tr} G_{i j}(i \omega)$,

where $G_{i j}$ denotes the mean field propagator in the presence of the impurities, and the trace is over lattice sites (and the inverse imaginary time derivative can be transformed into a frequency sum). The Gaussian fluctuation term is written in terms of the Bose fields as

$$
\begin{aligned}
& L_{G a u s s}=\frac{1}{2 \beta} \sum_{\omega}\left[i \lambda_{i}(\omega) i \lambda_{m}(\omega) S_{i m}^{\lambda \lambda}+\rho_{m}(\omega) i \lambda_{i}(\omega)\right. \\
& \left.+i \lambda_{m}(\omega) \rho_{i}(\omega) S_{i m}^{\lambda \rho}+\rho_{m}(\omega) \rho_{i}(\omega) S_{i m}^{\rho \rho}\right]
\end{aligned}
$$

where the Gaussian order Bose propagators are given by

$$
\begin{aligned}
S_{i m}^{\lambda \lambda}\left(\omega_{q}\right) & =\frac{1}{\beta} \sum_{\omega_{a}} G_{i m}\left(\omega_{a}\right) G_{i m}\left(\omega_{a}+\omega_{q}\right) \\
S_{i m}^{\lambda \rho}\left(\omega_{q}\right) & =\frac{1}{\beta} \sum_{\omega}\left[G_{i m}\left(\omega_{a}\right) G_{m p}\left(\omega_{a}+\omega_{q}\right) \rho t_{p i}+[i \rightarrow p]\right. \\
S_{i m}^{\rho \rho}\left(\omega_{q}\right)= & \frac{1}{\beta} \sum_{\omega_{a}}\left[G_{i m}\left(\omega_{a}\right) G_{n p}\left(\omega_{a}+\omega_{q}\right) t_{m n} t_{p i}\right. \\
& +[m \rightarrow p]+[p \rightarrow s]+[i \rightarrow s, p \rightarrow i]
\end{aligned}
$$

where the square brackets represent similar terms, apart from the indicated swapping of indices. So far, 
the impurity positions are still explicitly specified, and the configurational averages have yet to be carried out. Formally, the next step is to perform the disorder average, which we carry out by performing the Gaussian integral over the Bose fields and expanding the resulting free energy in a power series in the RPA bubbles

$$
\begin{aligned}
F & =\frac{1}{2 \beta} \sum_{\omega_{q}} \operatorname{Tr} \ln \left[\Pi_{0}+\Pi_{i j}\right] \\
& =\frac{1}{2 \beta} \sum_{\omega_{q}} \operatorname{TR}\left[\ln \Pi_{0}\right]+\sum_{n}\left[\left[\left(\Pi_{0}\right)^{-1} \Pi_{i j}\right]^{n} / n\right],
\end{aligned}
$$

where the $\Pi_{i j}$ refer to the RPA components of the Bose propagators in equation (1). Following the standard approach to calculating the free energy in an interacting disordered system [3] we replace the disorder average of $\Pi_{i j}^{n}$ by $\left\langle\Pi_{i j}\right\rangle^{n}$ in order to extract the dominant low energy diffusive corrections to the RPA bubbles. The next step is to perform the disorder average over the Bose propagators and we carry this out by keeping the ladder diagrams in the impurity potential (see Fig. 1). The free energy then becomes

$F=\frac{1}{2 \beta} \operatorname{Tr} \ln \left(\Pi_{0}+\left\langle\Pi_{i j}\right\rangle\right)$

and the Bose propagators have translational invariance restored and take the form:

$$
\begin{aligned}
& S_{\rho \rho}\left(q, \omega_{q}\right)=i \lambda-\frac{N}{\beta} \sum_{\omega_{q}} t_{k+q} G^{e x}\left(k, \omega_{a}\right) \\
& +N \frac{\rho_{2}}{2 \beta} \sum_{\omega_{a}}\left[I_{2}\left(q, \omega_{a}, \omega_{q}\right)+I_{1}\left(q, \omega_{a}, \omega_{q}\right)^{2} S\left(q, \omega_{a}, \omega_{q}\right)\right] \\
& S^{\lambda, \rho}\left(q, \omega_{q}\right)=i \rho\left[1+\frac{N}{2 \beta} \sum_{\omega_{a}} I_{1}\left(q, \omega_{a}, \omega_{q}\right)\right] \\
& \quad \times\left[1+n_{i} u(0)^{2} I_{0}\left(q, \omega_{a}, \omega_{q}\right) S\left(q, \omega_{a}, \omega_{q}\right)\right]
\end{aligned}
$$

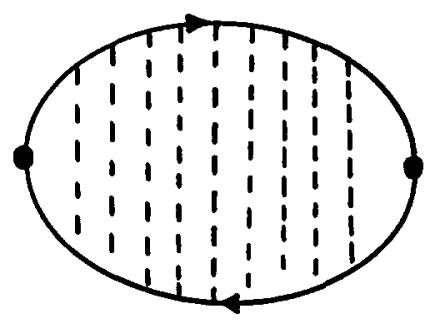

Fig. 1. Ladder diagrams in the impurity potential that contribute to the RPA components of the Bose propagators. The single lines denote the electron Green's functions with self energies given by the Born approximation in the impurity potential. A similar diagram can be drawn for the quantity $L_{i j k l}(E, \omega)$ that enters into the decay rate calculation.

$$
\begin{aligned}
& S^{\lambda \lambda}\left(q, \omega_{q}\right)=\frac{N}{2 \beta} \sum_{\omega_{a}} I_{0}\left(q, \omega_{a}, \omega_{q}\right) \\
& \times\left[1+n_{i} u(0)^{2} I_{0}\left(q, \omega_{a}, \omega_{q}\right) S\left(q, \omega_{a}, \omega_{q}\right),\right.
\end{aligned}
$$

where $G^{e x}$ denotes the exact quasiparticle Green's function appearing at the leading order in $1 / N$, averaged over the disorder. The $I_{n}$ are given by

$$
\begin{aligned}
I_{n}\left(q, \omega_{a}, \omega_{q}\right)= & \sum_{k}\left(t_{k}+t_{k+k_{1}}\right)^{n} G\left(k_{1}, \omega_{a}\right) \\
& \times G\left(k+k_{1}, \omega_{a}+\omega_{q}\right)
\end{aligned}
$$

and the quantity $S\left(q, \omega_{a}, \omega_{q}\right)$ is defined by

$$
\begin{aligned}
& S\left(q, \omega_{a}, \omega_{q}\right) \\
& \quad=\left[1-\frac{1}{2 \tau N^{*}(0)} \sum_{k} G\left(k+q, \omega_{a}+\omega_{q}\right) G\left(k, \omega_{a}\right)\right]^{-1} .
\end{aligned}
$$

The impurity effects are taken into account both in the ladder sum entering the above expression and in the self energy corrections to the electron Green's function;

$G\left(k, \omega_{a}\right)^{-1}=i \omega_{a}-\rho^{2} t_{k}-\frac{i}{2 \tau} \operatorname{sgn}\left(\omega_{a}\right)$,

where, as in the conventional weak disorder approaches, we keep only the lowest order Born approximation to the impurity self energy in the Green's functions that enter into the ladder sum. The density of states at the Fermi level is given by $N^{*}(0)$ and the quasiparticle lifetime is given by $1 /(2 \tau)=n_{i} u(0)^{2} N^{*}(0)$. Here $u(0)$ denotes the zero wavevector component of the impurity potential. Adopting the same Born approximation for the single particle Green's functions that enter into the mean field equations (5) and (6) leads to these taking the form

$$
\begin{aligned}
& i \lambda=\sum_{k} t_{k}\left[\frac{1}{2}-\frac{1}{\pi} \tan ^{-1}\left(2 \tau \rho_{0}^{2} t_{k}\right)\right] \\
& \rho^{2}=Q-\sum_{k}\left[\frac{1}{2}-\frac{1}{\pi} \tan ^{-1}\left(2 \tau \rho_{0}^{2} t_{k}\right)\right] .
\end{aligned}
$$

It can be seen from these equations that the Born approximation spreads out the Fermi surface discontinuity but otherwise leaves the mean field parameters unaffected (i.e. $\rho$ and $i \lambda$ are still real). It is known from Anderson lattice studies [8] that more sophisticated ways of performing the disorder average, such the CPA make only small quantitative changes in the mean field parameters. Within Born approximation the mean field equations are, apart 
from corrections of order $N^{*}(0) / \tau \ll 1$, identical to their values in the absence of disorder. Returning to the dynamic effects we evaluate the ladder sum contributions to the quantity $S\left(q, \omega_{a}, \omega_{q}\right)$ that enters the Bose propagators; for small $\omega_{q}, q$ we find

$$
\begin{aligned}
& S\left(q, \omega_{a}, \omega_{q}\right) \\
& \quad=\frac{\theta\left(\omega_{a}\right) \theta\left(-\omega_{a}-\omega_{q}\right)+\theta\left(-\omega_{a}\right) \theta\left(\omega_{a}+\omega_{q}\right)}{\left|\omega_{q}\right| \tau+D q^{2} \tau} .
\end{aligned}
$$

Here $\mathrm{D}$ denotes the diffusion constant $\nu_{F} \tau^{2} / d$ where $d$ is the dimensionality and $\nu_{F}$ the renormalized Fermi velocity. In the low wavevector and low energy limit $I_{n}=2 \pi \tau N^{*}(0)\left(2 t_{0}\right)^{n}$. After some manipulation we obtain for the Bose propagators

$$
\begin{aligned}
& S^{\rho \rho}\left(q, \omega_{q}\right)=N \frac{q^{2}}{2 m \beta} \sum_{w_{a}, k_{a}} G\left(k_{a}, \omega_{a}\right)+\left(2 t_{0} \rho\right)^{2} F\left(q, \omega_{q}\right), \\
& S^{\lambda \rho}\left(q, \omega_{q}\right)=\rho\left[1+2 t_{0} F\left(q, \omega_{q}\right)\right], \\
& S^{\lambda \lambda}\left(q, \omega_{q}\right)=F\left(q, \omega_{q}\right)=-N N^{*}(0) \frac{D q^{2}}{2\left|\omega_{q}\right| \tau+2 D q^{2} \tau},
\end{aligned}
$$

which defines $F\left(q, \omega_{q}\right)$. Here $t_{0}$ denotes the bare kinetic energy at the Fermi surface and we have used the mean field results to simplify the first two terms in $S^{\rho \rho}$. The $q^{2}$ term [the first term in equation (18)] can now be rewritten using the second mean field equation (averaged over disorder) in terms of the band filling.

Turning to the physical consequences of the above we note that the addition of the source field term coupled to the electron density allows us to derive in standard fashion the charge susceptibility

$\chi(q, \omega)=\rho^{2} \frac{S_{\lambda \lambda}(q, \omega)}{\operatorname{det} S(q, \omega)}$

which has a pole at the frequency $\omega_{q}$ defined as the zero of $\operatorname{det} S\left(q, \omega_{q}\right)$. On using the small $\omega$, small $q$ expansion for the Boson propagators given in equations (16), the numerator of $\operatorname{det} S(q, \omega)$ reduces, after analytic continuation of the frequency onto above the real axis, to a term proportional to

$i \omega-A D q^{2}-\alpha D \frac{q^{4}}{k_{F}^{2}}$

where $A=1+2 N\left|t_{0}\right| N^{*}(0)$ and $\alpha=(3 / 4) q^{2}$ $\left(\mathrm{m}^{*} / \mathrm{m}\right)^{2}$, and we have used the mean field results to write the $q^{2}$ term in $S^{\rho \rho}$ in terms in terms of the Fermion density $n$ and the mass enhancement $m^{*} / m=(1-n)^{-1}$. At low $q$ and for moderate fillings the usual diffusion pole behaviour is obtained, albeit with a modified diffusion constant, renormalised by the factor $A$. This factor plays the role of the Landau parameter in the weak interaction theories [3] and is reflected in the specific heat corrections at low temperatures, as we shall see.

However, the important feature of equation (20) lies in the behaviour of this mode at larger $q$ and close to $1 / N$ filling, since the $q^{4}$ term then becomes dominant. We note that for fillings close to $1 / N$ this $q^{4}$ term has replaced the usual diffusion pole. This type of "super-diffusive" mode has to our knowledge no classical analogy, except in elasticity theory. It may seem at first sight inconsistent to dwell on this $q^{4}$ term when we have neglected other $q^{4}$ terms in our derivation of the low wavevector, low energy behaviour of the Bose propagators in equation (2).

However, these "conventional" $q^{4}$ corrections arise on an energy scale set by $\tau^{-1}$ and on a momentum scale set by the diffusion constant $D$, and by comparing the above derived $q^{4}$ term with these conventional $D q^{4}\left(\nu_{F} \tau\right)^{2}$ terms we can see that it will win out when $m^{*} / m \gg \epsilon_{F} \tau \gg 1$. So for extremely large mass enhancements the above $q^{4}$ term will dominate over the diffusive $q^{4}$ terms, and will even dominate over the $q^{2}$ term for $q / k_{F}>m / m^{*}=\rho^{2}$. Hence asymptotically close to $1 / N$ filling the $q^{4}$ behaviour will take over from the usual $q^{2}$ form.

Particularly interesting consequences of the present theory concern the low temperature specific heat, which in the weakly interacting disorder theories is expected to show novel temperature dependences, depending on the dimensionality [3]. In contrast noninteracting system show localization effects only in their transport properties.

The free energy at Gaussian $1 / N$ order can then be written

$$
\begin{aligned}
& F=\frac{1}{2 \beta} \sum_{\omega} \ln \operatorname{det} S(q, \omega)=\frac{-1}{2 \pi} \sum_{q} \int \mathrm{d}(\omega) n(\omega) \\
& {\left[\tan ^{-1}\left(\frac{\omega}{A D q^{2}+\alpha D q^{4} / k_{F}^{2}}\right)-\tan ^{-1}\left(\frac{\omega}{D q^{2}}\right)\right],}
\end{aligned}
$$

where we have replaced the sum by an integral over the Bose function $n(\omega)$. For the case of moderate mass enhancements we obtain

$C \nu=-\rho_{3}\left[1-A^{-3 / 2}\right]\left(\frac{T}{D}\right)^{3 / 2}$

in three dimensions and

$C \nu=\rho_{2}\left[1-\frac{1}{A}\right] \frac{T}{D} \ln \left(\frac{T}{D}\right)$

in two dimensions. Here the constants are given by $p_{2}=\left(1 / 4 \pi^{2}\right) a(2), p_{3}=\left(1 / 2 \pi^{2}\right) a(5 / 2) b(0,4)$. These 
terms agree with the power law behaviour obtained in weakly interacting theories [3] with the difference that $A$ enters instead of the usual Landau parameters. The functional agreement with earlier works in the small filling, moderate mass enhancement regime is gratifying and to be expected.

The situation changes as we approach the $1 / N$ fractional filling limit. This time the $q^{2}$ terms may be neglected and the total specific heat results are instead

$C_{\nu}=q_{3}\left(\frac{T}{D \alpha}\right)^{3 / 4}-p_{3}\left(\frac{T}{D}\right)^{3 / 2}$

in three dimensions where $q_{3}=0.41$ and

$$
C_{\nu}=-q_{2}(T / \alpha D)^{1 / 2}-p_{2} T \ln (T / D)
$$

in two dimensions where $q_{2}=0.78$. Thus at sufficiently low temperatures, near the critical metalinsulator filling, the specific heat develops an additional sublinear fractional power law dependence, demonstrating an even stronger dominance of the disorder contributions over the conventional linear temperature dependence, even for weak disorder.

Turning now to the quasiparticle decay rate we first have to resolve a question as to its definition. Within the $k$-state description it is not possible to refer to quasiparticles when the Born self-energy already has such an elastic contribution, as. in equation (14). The way out, as shown by Abrahams et al. [14] (see also [3]) is to start with the correct eigenstates of the non-interacting disordered quasiparticle problem-in our case these are the eigenstates of the disordered mean field problem, which we shall denote by $\Psi_{\alpha}(i)$. The original Fermion states can be expressed in terms of these states and their creation operators $a_{\alpha, m}^{+}$, as follows

$f_{i, m}^{+}=\sum_{\alpha} a_{\alpha, m}^{+} \Psi_{\alpha}^{*}(i)$.

Performing the trace over the new Fermion operators leads only to a modification of the effective Fermion propagator [the argument of the logarithm in equation (4)] which in the new representation becomes

$A_{\alpha, \beta}\left(i \omega_{a}, i \omega_{b}\right)=A_{\alpha, \beta}^{0}\left(i \omega_{a}, i \omega_{b}\right)+X_{\alpha, \beta}(a, b)$

where the now diagonal mean field propagator is given by the inverse of

$A_{\alpha, \beta}\left(i \omega_{a}\right)=\sum_{i, j} \Psi_{\alpha}^{*}(i)\left[\left(i \omega_{a}-\mu+i \tilde{\lambda}_{j}\right) \delta_{i, j}+t_{i, j} p^{2}\right] \Psi_{\beta}(j)$ and the fluctuations are obtained from

$$
\begin{aligned}
& X_{\alpha, \beta}(a, b) \\
& =\sum_{i, j} \Psi_{\alpha}^{*}(i)\left[i \lambda_{i}\left(\omega_{a}-\omega_{b}\right) \delta_{i, j}+t_{i, j} \rho\left[\left(\rho_{i}\left(\omega_{a}-\omega_{b}\right)\right.\right.\right. \\
& \left.\quad+\rho_{j}\left(\omega_{a}-\omega_{b}\right)\right] \\
& \quad+t_{i, j} \frac{1}{\beta} \sum_{\omega_{q}} \rho_{i}\left(\omega_{a}-\omega_{b}-\omega_{q}\right) \rho_{j}\left(\omega_{q}\right) \Psi_{j}(j) .
\end{aligned}
$$

The fact that the mean field propagator is now diagonal in the new representation means that we can extract a contribution to the Green's function from the Gaussian fluctuations which takes the form of a self energy

$$
\begin{aligned}
& \Sigma_{\epsilon}\left(i \omega_{a}\right)=\int D \rho D \lambda e^{\left(-\int d \tau L(\tau)\right.} T \\
& \quad \times \Sigma_{\delta, c} \epsilon, \delta(a, c) A_{\delta}^{0-1}(c) X_{\delta . \epsilon}(c, a),
\end{aligned}
$$

although we are in fact only really interested in the impurity averaged version of this

$\Sigma_{E}(i \omega)=\frac{1}{N^{*}(0)} \sum_{\epsilon}\left\langle\delta\left(E-E_{\epsilon}\right) \Sigma_{\epsilon}(i \omega)\right\rangle$.

We now follow Abrahams et al. [14] and perform the disorder average separately in (a) the exponential part, which give rise to the Gaussian Bose propagators discussed earlier, and (b) the non-exponential component of equation (30). The latter is simply evaluated using the fact that equation (28) contains a combination of four eigenstates, one inverse propagator and a delta function. This combination may be related to the quantity

$L_{i j k l}\left(E, \omega_{c}\right)=\frac{1}{2 i \pi}\left\langle\left[G_{i l}^{A}(E)-G_{i l}^{R}(E)\right] G_{k j}\left(\omega_{c}\right)\right\rangle$,

where $R(A)$ refers to the retarded (advanced) part of the leading order Green's function. The impurity average over the exponential gives a translationally invariant Gaussian functional form to the exponential, so that the self energy becomes

$$
\begin{aligned}
& \Sigma_{E}\left(i \omega_{a}\right)=\frac{1}{\beta} \sum_{i k \omega_{c}} D \rho D \lambda\left\{L_{i i k k}\left(E, \omega_{c}\right) i \lambda_{i}(a-c) i \lambda_{k}(c-a)\right. \\
& \quad+\left[\left(L_{i j k l}\left(E, \omega_{c}\right)+(i \rightarrow j)\right] t_{i j} \rho \rho_{i}(a-c) i \lambda_{k}(c-a)\right. \\
& \quad+\left[L_{i i k l}\left(E, \omega_{c}\right)+(l \rightarrow k)\right] t_{k l} \rho_{i} \lambda_{i}(a-c) \rho_{k}(c-a) \\
& \quad+\left[L_{i j k l}\left(E, \omega_{c}\right)+(i \rightarrow j)+(j \rightarrow k, k \rightarrow l, l \rightarrow k)\right. \\
& \quad+(i \rightarrow k, j \rightarrow i, k \rightarrow l, l \rightarrow k)] \\
& \left.\quad \times t_{i j} t_{k l} \rho^{2} \rho_{i}(a-c) \rho_{k}(c-a)\right\} \exp \left(-\int \mathrm{d} \tau L(\tau)\right) Z .
\end{aligned}
$$


The terms in braces refer to appropriate permutations of the suffices of $L_{i j k l}\left(E, \omega_{c}\right)$, and the functional integral over the Bose fields still has to be carried out. The crucial aspect of this calculation lies in the fact that $L_{i j k l}(E, \omega)$ is itself related to a density-density correlation function, via the ensemble average of two Green's functions. This results, upon performing the disorder average over these two Green's functions, in a ladder-type summation leading again to a diffusion pole result for $L_{i j k l\left(z, \omega_{c}\right)}$. The final result is that the self energy takes the form

$$
\begin{aligned}
& \Sigma_{E}\left(i \omega_{a}\right)=\frac{1}{2 \beta} \sum_{q, \omega_{q}} 2 \pi N^{*}(0) \tau \operatorname{disc}_{z} S\left(q, z, \omega_{a}-\omega_{q}\right) \\
& \quad \times\left[S_{\lambda \lambda}^{-1}\left(q, \omega_{q}\right)+4 t_{0} S_{\lambda \rho}^{-1}\left(q, \omega_{q}\right)+\left(2 t_{0} \rho\right)^{2} S_{\lambda \lambda}^{-1}\left(q, \omega_{q}\right)\right] .
\end{aligned}
$$

where

$\operatorname{disc}_{z} f(z)=\frac{1}{2 i \pi}[f(\epsilon+i \delta)-f(\epsilon-i \delta)]$

Using the low $\omega, q$ form of the Bose propagators leads to the following form for the self energy

$\Sigma_{E}\left(i \omega_{a}\right)=$

$\sum_{q, \omega_{q}} \frac{-2 i T t_{0}(q) N^{*}(0)\left|\omega_{q}+D q^{2}\right|}{\left[\omega_{q}+A(q) D q^{2}\right]\left[\omega_{a}-\omega_{q}+i E+D q^{2} \operatorname{sgn}\left(\omega_{a}-\omega_{q}\right)\right]}$,

where $A(q)=1+2 N^{*}(0) N\left|t_{0}(q)\right|$, with $t_{0}(q)=t_{0}$ $n_{f} q^{2} / 8 m \rho^{2}$. Note the appearance of two diffusion like terms in the denominator. The sum over frequency may now be converted into an integral over Bose functions and the zero temperature limit can be taken with the result that on the mass-shell $(E=\omega)$, where we have analytically continued $\omega_{a}$ onto the real axis, we obtain for the imaginary part of the self energy

$$
\begin{aligned}
\operatorname{Im} \Sigma_{\omega}(\omega)= & \frac{N^{*}(0)}{2 \pi} \sum_{q} \frac{t_{0}(q)}{1+A(q)}\left[\ln \left(1+\frac{\omega^{2}}{\left(A D q^{2}\right)^{2}}\right)\right. \\
& \left.-\ln \left(1+\frac{\omega^{2}}{D^{2} q^{2}}\right)\right]
\end{aligned}
$$

and we proceed to analyse this expression in the limits of moderate fillings and close to the metal-insulator transition. For moderate fillings we may replace $t_{0}$ and $A$ by their low wavevector limits, and perform the simple integral to obtain

$\operatorname{Im} \Sigma(\omega+i \delta)=\frac{a d}{D^{d / 2}}\left[\frac{1}{A^{d / 2}}-1\right] \omega^{d / 2} \frac{A_{0}^{s}}{2-A_{0}^{s}}$, where

$a=\frac{1}{4 \pi N} S_{d} \frac{\pi}{d} 2^{(d-2) / 2}$

and $S_{d}$ is the solid angle, for $d=2$ or 3 . This form is identical to that in weak interaction theories [15] except that we have a first principles calculation of the micropic parameter

$$
A_{0}^{s}=\frac{2 N N^{*}(0)\left|t_{0}\right|}{1+2 N N^{*}(0)\left|t_{o}\right|}
$$

On the other hand, close to the critical metalinsulator filling the $q^{2}$ components of $t_{0}(q), A(q)$ dominate, and we obtain

$\operatorname{Im} \Sigma(\omega+i \delta)=\frac{S_{d}}{4 \pi N}\left[f(d, 8) \frac{\omega^{d / 4}}{\tilde{D}}-f(d, 4) \frac{\omega^{d / 2}}{D}\right]$

where $\tilde{D}=\alpha D / k_{F}^{2}$, with $\alpha$ defined after equation (18), and we have introduced the constants

$f(d, n)=\int_{0}^{\infty} \mathrm{d} q q^{d-1} \ln \left[1+q^{-n}\right]$.

Thus, in addition to the expected $\omega^{d / 2}$ behaviour we also obtain a $\omega^{d / 4}$ component at low energies.

In conclusion, we have studied the effects of weak disorder on the $U=\infty$ Hubbard model, using the $1 / N$ expansion to treat the strong correlation effects, and summing ladder graphs in the impurity potential. For fillings away from the critical value, the usual diffusion pole behaviour is found in the density correlation function, with the infinite $U$ effects reflected in a Gutzwiller type mass enhancement. The $1 / N$ contributions to the decay rate and the specific heat show the same temperature dependence as found in previous weak interaction approaches. However, as the filling approaches its critical value, the diffusion pole behaviour is modified, the $q^{2}$ term being replaced by a $q^{4}$ contribution. The specific heat at the $1 / N$ level now shows sublinear temperature dependences $\left(T^{3 / 4}\right.$ and $T^{1 / 2}$ in $3 D$ and $\left.2 D\right)$ while the decay rate acquires a $\omega^{d / 4}$ component.

Thus, we have shown, using a combination of standard weak disorder techniques, combined with recently developed approaches to the strong correlation problem, that novel non Fermi liquid corrections can be found to the decay rate and specific heat in disordered strongly interacting electron systems. Extensions of the present work to the $t-J$ model and Anderson lattice are planned. 


\section{REFERENCES}

1. P. Scoboria, J.E. Crow \& T. Mihalisin, J. Appl. Phys. 50, 1895 (1979); J.M. Lawrence, J.D. Thompson \& Y.Y. Chen, Phys. Rev, Lett. 54, 2537 (1985); M.C. Aronson, J.D. Thompson, J.L. Smith, Z. Fisk \& M.W. McElfresh, Phys. Rev. Lett. 63, 2311 (1989).

2. B. Alekashin et al., Sov. Phys. JETP 68, 382 (1989).

3. P.A. Lee \& T.V. Ramakrishnan, Rev. Mod. Phys. 57, 287 (1985).

4. C. Di Castra, Anderson Localisation: Proc. Int. Symp., Tokyo, Jpn (Edited by T. Ando \& H. Fukuyama), Springer, Berlin (1982).

5. F.J. Wegner, Anderson Localisation, Proc. Int. Symp., Sanda-Shi, Jpn (Edited by Y. Nagaoka \& H. Fukuyama), Springer, Berlin (1982).

6. G. Zimanyi \& E. Abrahams, Phys. Rev. Lett. 64, 2719 (1990).
7. A.M. Finkelstein, Sov. Phys. JETP 57, 97 (1985).

8. Z. Tesanovich, Phys. Rev. B34, 5212 (1986); Wang $\mathrm{Xu} \&$ Zheng-Zhong Li; J. Phys. CM2, 109 (1990); R. Freytag \& J. Keller, Z. Phys. B80, 241 (1990).

9. R. Sollie \& P. Schlottmann, Phys. Rev. B42, 6099 (1990).

10. J.W. Rasul Phys. Rev. B44, 11802 (1991).

11. G. Kotliar \& J. Liu, Phys. Rev. Lett. 61, 1784 (1988).

12. W.N. Huang \& J.W. Rasul, Phys. Rev. B45, 3995 (1992).

13. N. Read \& D.M. Newns, J. Phys. C16, 3273 (1983).

14. E. Abrahams, P.W. Anderson, P.A. Lee \& T.V. Ramakrishnan, Phys. Rev. B24, 6783 (1981).

15. B.L. Altshuler \& A.G. Aronov, Solid State Commun. 46, 429 (1983). 